\title{
Colored scalar mediated nucleon decays to an invisible fermion
}

\author{
Svjetlana Fajfer@* and David Susič $\circledast^{\dagger}$ \\ Jožef Stefan Institute, Jamova 39, 1000 Ljubljana, Slovenia \\ and Faculty of Mathematics and Physics, University of Ljubljana, Jadranska 19, 1000 Ljubljana, Slovenia
}

(Received 26 October 2020; accepted 6 February 2021; published 18 March 2021)

\begin{abstract}
We investigate nucleon decays to light invisible fermion mediated by the colored scalar $\bar{S}_{1}=(\overline{3}, 1,-2 / 3)$ and compare them with the results coming from the mediation of $S_{1}=(\overline{3}, 1,1 / 3)$. In the case of $\bar{S}_{1}=(\overline{3}, 1,-2 / 3)$ uplike quarks couple to the invisible fermion, while in the case of $S_{1}=(\overline{3}, 1,1 / 3)$ the downlike quarks couple to the invisible fermion. For the mass of invisible fermion smaller than the mass $m_{p}-m_{K}$, proton (neutron) can decay to $K$ and invisible fermion and the masses of $\bar{S}_{1}$ and $S_{1}$ are in the region $\sim 10^{15} \mathrm{GeV}$. The decays of nucleons to pions and invisible fermion can occur at the tree level, but in the case of $\bar{S}_{1}$ they come from a dimension-nine operator and are therefore suppressed by several orders of magnitude compared to the decays into kaons. For the invisible fermion mass in the range (937.8, 938.8 MeV), decay of neutron $n \rightarrow \chi \gamma$ induced by $\bar{S}_{1}$ is possible at the loop level, while the proton remains stable. The branching ratio of such decay is $\leq 10^{-6}$, which does not explain neutron decay anomaly, but is in agreement with the Borexino experiment bound. We comment on low-energy processes with the nucleonlike mass of $\chi$ in the final state as $\Lambda \rightarrow \chi \gamma$ and heavy hadron decays to invisibles.
\end{abstract}

DOI: $10.1103 /$ PhysRevD.103.055012

\section{INTRODUCTION}

Many constraints on physics beyond the Standard Model (SM) at low-energies are already well established. Although, it seems that possibilities for new physics (NP) at low energies are known and well studied, there are some chances that light neutral particles may have evaded experiments due to their long lifetime. Recently, the author of Ref. [1] suggested this possibility and investigated a number of scenarios with light fermions carrying lepton or baryon number. In this work we focus on the light fermions carrying baryon number. As already summarized by many authors [1-12], such interactions between quarks and right-handed fermions are mediated by colored scalars. Obviously, colored scalars can couple either to downlike quarks or to uplike quarks depending on their charge $-1 / 3$ or $2 / 3$. On the experimental side, the KamLAND Collaboration [13] has already searched for the invisible decays of neutrons but assumed a zero mass of the invisible state.

Leptoquarks mediate SM quark and lepton interactions. In the case where instead of a lepton there is a fermion with

\footnotetext{
svjetlana.fajfer@ijs.si

david.susic@ijs.si
}

Published by the American Physical Society under the terms of the Creative Commons Attribution 4.0 International license. Further distribution of this work must maintain attribution to the author(s) and the published article's title, journal citation, and DOI. Funded by SCOAP ${ }^{3}$. quantum numbers of a right handed neutrino, we name the mediator colored scalar. Following the notation of [14], we present in Table I colored scalars which have interactions with a such state as well as the diquark interactions. The scalar $S_{1}$ couples to leptons and therefore plays a role of a leptoquark. Contrary to $S_{1}(\overline{3}, 1,1 / 3), \bar{S}_{1}(\overline{3}, 1,-2 / 3)$ is a colored-scalar (triplet of color group, singlet of weak, with hypercharge and electric charge equal to $2 / 3$; here the weak hypercharge $Y$ is defined as $Q=I_{3}+Y$ ). Due to its quantum numbers, $S_{1}$ might have interactions with SM doublets, quarks and leptons, while the colored scalar $\bar{S}_{1}=$ $(\overline{3}, 1,-2 / 3)[14,15]$ only has two type of interactions with right-handed fermions. One with up quarks and with neutral weak right-handed singlets and the second one is an interaction between different generations of the down quarks [14].

In addition to the general study of Ref. [1], an interesting possibility was discussed in the literature with the main concern being stability of proton, while neutron or hydrogen atom are unstable $[2-4,16]$. For example, the authors of [17] pointed out that there is a discrepancy between the neutron lifetime measured in beam and bottle experiments. This idea initiated new experimental studies that supported discrepancy between the two experimental results [18] on the level of 3.6 $\sigma$. The world average of the bottle experiment according to PDG [19] is $\tau_{n}^{\text {bottle }}=(880.2 \pm$ 1.0) s and $\tau_{n}^{\text {beam }}=(888.0 \pm 2.0)$ s. In Ref. [17] this discrepancy was addressed by assuming that a neutron can decay to dark matter (DM) and one photon, or two types of 
TABLE I. The colored scalars $\bar{S}_{1}$ and $S_{1}$ interactions with invisible fermions and two quarks. Here we use only right-handed couplings of $S_{1}$. Indices $i, j$ refer to quark generations.

\begin{tabular}{lcc}
\hline \hline Colored scalar & Invisible fermion & Diquark \\
\hline$S_{1}=(\overline{3}, 1,1 / 3)$ & $\bar{d}_{L}^{C}{ }^{i} \nu_{R} S_{1}$ & $\bar{u}_{R}^{C i} d_{R}^{j} S_{1}^{*}$ \\
$\bar{S}_{1}=(\overline{3}, 1,-2 / 3)$ & $\bar{u}_{R}^{C}{ }^{j} \nu_{R} \bar{S}_{1}$ & $\bar{d}_{R}^{C}{ }^{i} d_{R}^{j} \bar{S}_{1}^{*}$ \\
\hline \hline
\end{tabular}

DM. In order to avoid proton destabilization, the authors of this proposal suggested that the dark fermion should have mass in the range $m_{p}-m_{e} \leq m_{\chi} \leq m_{p}+m_{e}$ (or $937.8 \mathrm{MeV}<m_{\chi}<938.8 \mathrm{MeV}$ ) in the case of neutron decay to DM fermion and $\gamma$, while the photon energy is in the range $0.782 \mathrm{MeV}<E_{\gamma}<1.664 \mathrm{MeV}$. The branching ratio for the decay $n \rightarrow \chi \gamma$ which explains the neutron lifetime anomaly should be $\sim 10^{-2}$. The selection of this narrow mass window enables the DM to remain stable. Unfortunately, the direct search for the $n \rightarrow \chi \gamma$ decay at the level required to explain the neutron lifetime anomaly was unsuccessful [20]. Another possibility for the DM presence in the nucleon dynamics was offered in [21] in which the neutron can convert into mirror neutron, its dark partner from parallel mirror sector.

The approach of [17] assumes that a state with quantum numbers of $S_{1}=(\overline{3}, 1,1 / 3)$ mediates this interaction.

The fermionic dark matter in this approach is a color weak singlet, neutral state $(1,1,0)$, which can couple to downlike quarks. Recently, the authors of [2] questioned a possibility that a hydrogen atom is unstable, whereas a proton remains stable. They considered a case where the photon is emitted with the energy smaller than the nucleon binding energy inside nucleus. They noticed that the results of the Borexino experiment [22] allow the threshold for the detection of electromagnetic energy depositions to be reduced down to $\sim 200 \mathrm{keV}$. Using Borexino data [22] they found out that nontrivial constraints arise from the subdominant radiative decay mode. In such a way, we obtain a direct test of scenarios where the neutron mixes with an invisible fermion without the nuclear physics complications. The main message of this study is that Borexino data restrict the branching ratio of the $n \rightarrow \chi \gamma$ to be smaller than $10^{-4}$. The existence of heavy neutron stars also gives the strong limits, since $n \rightarrow \chi \gamma$ would allow neutron stars to reach masses below the observed ones [2326]. The color scalar or vector mediation in the processes of interactions of the DM with the SM fermions were considered in varieties of the models (see, e.g., [27-31]). The new invisible fermion is stable and therefore might be a candidate for the DM. For the kinematic mass of $m_{\chi} \leq m_{p}$ only DM annihilation channel $\chi \chi \rightarrow u_{i}^{c} u_{j}^{c}$ is allowed. This has been widely discussed in literature [27,29,32-34]. However, the calculated value is smaller than the thermal cross section for DM $3 \times 10^{-26} \mathrm{~cm}^{3} / \mathrm{s}^{2}$ [32]. Such a result means that thermal freeze-out leads to over-produced DM and possible scenarios of a nonthermal production mechanism are necessary to explain the observed DM abundance [32]. Since we consider only phenomenological aspects of the invisible fermion couplings to a colored scalar and one of the up quarks, we use invisible fermion instead of DM fermion.

In this paper we first write down Lagrangians for $\bar{S}_{1}$ and $S_{1}$ in Sec. II. Then in Sec. III we consider decays of nucleons $p, n \rightarrow K \chi$ which can occur at tree level, as well as $p, n \rightarrow \pi \chi$. We compare our results with results coming from the mediation of $S_{1}$. In Sec. IV we discuss decay $n \rightarrow \chi \gamma$ due to mediation of $\bar{S}_{1}$. Section V contains a discussion of consequences at low energies. In Sec. VI we summarize our results.

\section{INTERACTIONS OF $\bar{S}_{1}$ AND $S_{1}$}

The Lagrangian describing $\bar{S}_{1}=(\overline{\mathbf{3}}, \mathbf{1},-2 / 3)$ interactions is

$$
\mathcal{L}_{\bar{S}_{1}} \supset+\bar{y}_{1 i j}^{\overline{R R}} \bar{u}_{R}^{C i} \bar{S}_{1} \chi^{j}+\bar{z}_{1 i j}^{R R} \bar{d}_{R}^{C i} \bar{S}_{1}^{*} d_{R}^{j}+\text { H.c. }
$$

This color scalar does not couple to charged leptons and interacts only with two different down quarks. In principle, in this Lagrangian three species of invisible fermions $\chi^{j} \equiv$ $(1,1,0)$ can exist with the quantum number of the righthanded neutrino $\nu_{R}$. In order to simplify the model, we assume that there is only one $\chi \equiv \chi^{j}$ for $j=1,2,3$ which can couple to the $u, c$ and $t$ quarks. In the matrix $\bar{y}_{1 i j}^{\overline{R R}}$ we then set $j=1$. Strictly speaking, the Lagrangian refers to quarks and invisible fermions in the flavor basis. In order to get these fields in the mass basis, one has to perform appropriate rotations (see for details [35]). Since we consider Lagrangians with the right-handed fields only, we treat our couplings in (1), as they are already in the mass basis. The color indices are not presented in (1).

Note that $\bar{z}_{1 i j}^{R R}$ is an antisymmetric matrix in any flavor basis, as well as in color indices (not specified here, but knowing that $\bar{d}_{R}^{C}{ }^{i} \bar{S}_{1}^{*} d_{R}^{j} \rightarrow \epsilon_{\alpha \beta \gamma} \bar{d}_{R, \alpha}^{C i} d_{R, \beta}^{j} \bar{S}_{1, \gamma}^{*}$ and $\left.\bar{z}_{1 i j}^{R R}=-\bar{z}_{1 j i}^{R R}\right)$.

In some proposals $\chi^{j}$ is considered to be a Majorana fermion whose mass can be introduced by the mass term $m_{\chi} \bar{\chi}^{c} \chi$. In such scenarios one can simply assign baryon number $B=2 / 3$ to $\bar{S}_{1}$ and $B=+1$ to $\chi$ [34]. That means then that the interacting Lagrangian preserves baryon number, while only the Majorana mass term will be source of the baryon number violation.

The full Lagragian for $S_{1}$ is given in Eq. (9) of [14]. Here we give only two terms of it, which we later use in our calculations

$$
\mathcal{L}_{S_{1}} \supset y_{1 i j}^{\overline{R R}} \bar{d}_{R}^{C}{ }^{i} S_{1} \chi^{j}+z_{1 i j}^{R R} \bar{u}_{R}^{C}{ }^{i} S_{1}^{*} d_{R}^{j}+\text { H.c. }
$$


Note that the last term can come with the opposite chirality too, which is not the case with $\bar{S}_{1}$.

\section{NUCLEON DECAYS TO PSEUDOSCALAR MESON AND INVISIBLE FERMION AT GUT SCALE}

In [1] the author considers a number of cases with the invisible fermion having nonzero lepton or baryon number. The most general Lagragnian with $\chi$ having baryon number $B=1$ can be written as $[1,36]$

$$
\mathcal{L}_{\chi}=\bar{\chi}\left(i \not \supset-m_{\chi}\right) \chi+\left(\frac{u_{i} d_{j} d_{k} \chi_{L}^{c}}{\Lambda_{i j k}^{2}}+\frac{Q_{i} Q_{j} d_{k} \chi_{L}^{c}}{\tilde{\Lambda}_{i j k}^{2}}+\text { H.c. }\right) .
$$

Here $\Lambda$ and $\tilde{\Lambda}$ denote the scales of NP. We use here notation introduced in [1] and only write the flavor indices, not indicating Lorentz, color, and isospin indices. Assuming baryon number conservation, neutron-anti-neutron oscillations do not occur.

Integrating out the leptoquark states $S_{1}$ or $\bar{S}_{1}$, one can straightforwardly write the effective Lagrangian for the $\left(u^{j}, d^{k}, d^{l}, \chi\right)$ interaction (see Figs. 1, 2, 3, and 4)

$$
\mathcal{L}_{\text {eff }}\left(\bar{S}_{1}\right)=\frac{\bar{y}_{1 j 1}^{\overline{R R}} \bar{z}_{1 k l}^{R R}}{M_{\bar{S}_{1}}^{2}} \epsilon_{\alpha \beta \gamma}\left(\bar{\chi}^{C} P_{R} u_{\alpha}^{j}\right)\left(\bar{d}_{\beta}^{C k} P_{R} d_{\gamma}^{l}\right) .
$$

In the case of $S_{1}$ one finds

$$
\mathcal{L}_{\text {eff }}\left(S_{1}\right)=\frac{y_{11 j}^{\overline{R R}} z_{1 k l}^{R R}}{M_{S_{1}}^{2}} \epsilon_{\alpha \beta \gamma}\left(\bar{\chi}^{C} P_{R} d_{\alpha}^{j}\right)\left(\bar{u}_{\beta}^{C k} P_{R} d_{\gamma}^{l}\right) .
$$

In Eqs. (4) and (5) the dimension-six operators are of the type $u_{i} d_{j} d_{k} \chi_{L}^{c}$ in Eq. (3). The last term in Eq. (3) can be

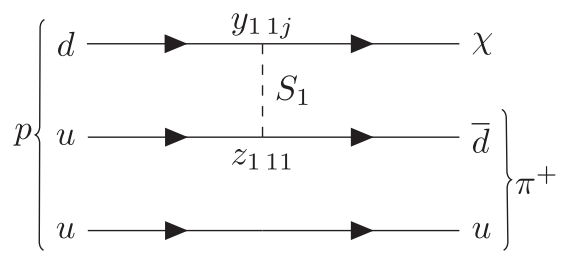

FIG. 1. The amplitude for $p \rightarrow \chi \pi^{+}$, induced by $S_{1}$.

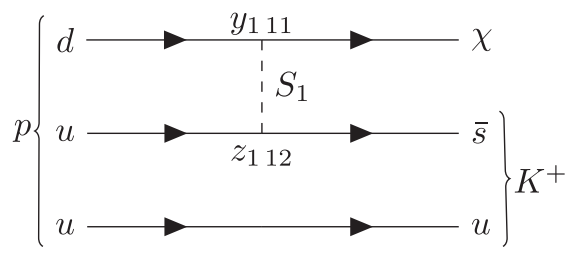

FIG. 2. The amplitude for $p \rightarrow \chi K^{+}$, induced by $S_{1}$.

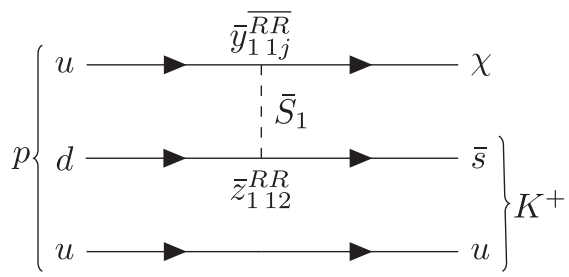

FIG. 3. Proton decay $p \rightarrow K^{+} \chi$, induced by $\bar{S}_{1}$.

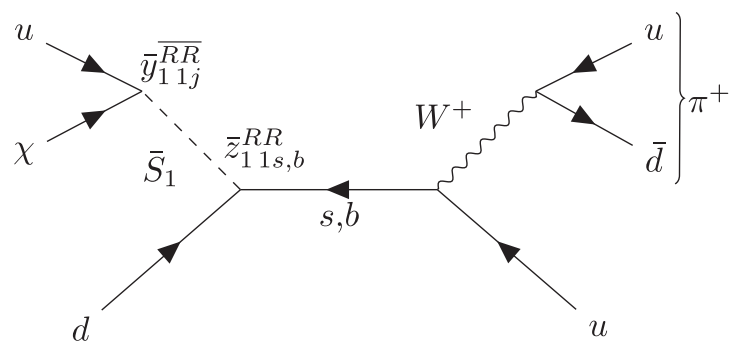

FIG. 4. Proton decay $p \rightarrow \pi^{+} \chi$ induced by dimension-nine operator due to $\bar{S}_{1}$ interaction.

generated only from the $S_{1}$ interactions with the left-handed quarks.

In order to obtain matrix elements of the operator between nucleon and pseudoscalar states one can use notation of Ref. [37]

$$
\begin{aligned}
& \left\langle P(p)\left|\epsilon_{\alpha \beta \gamma}\left(u_{\alpha}^{T} C P_{\Gamma} d_{\beta}\right) P_{\Gamma^{\prime}} s_{\gamma}\right| N(P, s)\right\rangle \\
& =P_{\Gamma}\left(W_{0}^{\Gamma \Gamma^{\prime}}\left(q^{2}\right)-i q W_{1}^{\Gamma \Gamma^{\prime}}\left(q^{2}\right)\right) u_{N}(P, s),
\end{aligned}
$$

with $W_{i}\left(q^{2}\right)$ being form-factors determined by lattice QCD. One can easily calculate $\bar{S}_{1}$ mediated decay amplitudes for $p \rightarrow K^{+} \chi$

$-i \mathcal{M}=-\frac{i \bar{y}_{11 j}^{R R} \bar{z}_{112}^{R R}}{m_{\bar{S}_{1}}^{2}} W_{0}^{R R}\left(k_{2}^{2}\right) \bar{u}_{\chi}\left(k_{2}, s_{2}\right) P_{R} u_{p}\left(k_{1}, s_{1}\right)$

with the decay width

$$
\begin{aligned}
\Gamma(p & \left.\rightarrow K^{+} \chi\right)=\frac{1}{32 \pi}\left(\frac{\bar{y}_{111}^{\overline{R R}} \bar{z}_{112}^{R R}}{m_{\bar{S}_{1}}^{2}}\right)^{2}\left|W_{0}^{R R}\right|^{2} \\
& \times \frac{m_{p}^{2}-m_{K^{+}}^{2}+m_{\chi}^{2}}{m_{p}^{3}} \lambda^{1 / 2}\left(m_{K^{+}}^{2}, m_{p}^{2}, m_{\chi}^{2}\right)
\end{aligned}
$$

and the decay width for $n \rightarrow K^{0} \chi$

$$
\begin{aligned}
\Gamma(n & \left.\rightarrow K^{0} \chi\right)=\frac{1}{32 \pi}\left(\frac{\bar{y}_{111}^{\overline{R R}} \bar{z}_{112}^{R R}}{m_{\bar{S}_{1}}^{2}}\right)^{2}\left|W_{0}^{R R}\right|^{2} \\
& \times \frac{m_{n}^{2}-m_{K^{0}}^{2}+m_{\chi}^{2}}{m_{n}^{3}} \lambda^{1 / 2}\left(m_{K^{0}}^{2}, m_{n}^{2}, m_{\chi}^{2}\right),
\end{aligned}
$$


where $\lambda(a, b, c)=a^{2}+b^{2}+c^{2}-2(a b+a c+b c)$. We use results $W_{0 p \rightarrow \pi^{+}}^{R R}=0.122 \mathrm{GeV}^{2}, \quad W_{0 p \rightarrow K^{+}}^{R R}=-W_{0 n \rightarrow K^{0}}^{R R}=$ $-0.085 \mathrm{GeV}^{2}$ [37,38]. For the intermediate $S_{1}$ one can use above results by making the replacements $\bar{z}_{112}^{R R} \rightarrow z_{112}^{R R}, m_{\bar{S}_{1}} \rightarrow m_{S_{1}}$. Experimental results on nucleon decays to invisible fermions only exist for invisible fermion with the negligible mass. The bounds on the lifetimes are $\tau\left(p \rightarrow \pi^{+} \nu\right)>\left(390 \times 10^{30}\right)$ yr [13], $\tau\left(n \rightarrow \pi^{0} \nu\right)>$ $\left(1100 \times 10^{30}\right) \mathrm{yr} \quad[13], \quad \tau\left(p \rightarrow e^{+} \nu \nu\right)>\left(170 \times 10^{30}\right) \mathrm{yr}$ [39]. As pointed out by the author of [1], these limits push the scale $m_{\bar{S}_{1}}$ above $10^{15} \mathrm{GeV}$. For the nucleon decays to pion and invisible fermion induced by $S_{1}$ one can use (9), replacing $m_{K} \rightarrow m_{\pi}, z_{112} \rightarrow z_{111}$. However, the decay amplitude $N \rightarrow \pi \chi$ induced by $\bar{S}_{1}$ can occur at loop level or it can appear at tree level, due to the operator of dimension nine, as explained in detail in [35]. In Fig. 4 the basic decay mechanism caused by the operator of dimension-nine is presented. The effective Lagrangian created by such transition is

$$
\begin{aligned}
\mathcal{L}_{9}= & \frac{8 G_{F}}{\sqrt{2}} \frac{\bar{y}_{11}^{\overline{R R}} \bar{z}_{112}^{R R}}{m_{\bar{S}_{1}}^{2}} \frac{V_{u d} V_{u s}^{*}}{m_{s}} \\
& \times \epsilon_{\alpha \beta \delta}\left(\bar{u}_{\zeta} P_{R} d_{\alpha}\right)\left(\bar{u}_{\beta}^{C} P_{L} d_{\zeta}\right)\left(\bar{\chi} P_{R} u_{\delta}\right),
\end{aligned}
$$

resulting in the amplitude

$$
\begin{aligned}
\mathcal{M}_{p \rightarrow \pi^{+} \chi}= & i \frac{4 G_{F}}{\sqrt{2}} \frac{\bar{y}_{11 j}^{\overline{R R}} \bar{z}_{112}^{R R}}{m_{\bar{S}_{1}}^{2}} \frac{V_{u d} V_{u s}^{*}}{m_{s}} \\
& \times \frac{f_{\pi^{+}} m_{\pi^{+}}^{2}}{\left(m_{u}+m_{d}\right)} \alpha_{L} \bar{u}_{\chi} P_{R} u_{p}
\end{aligned}
$$

and the decay width

$$
\begin{aligned}
\Gamma\left(p \rightarrow \pi^{+} \chi\right)= & \frac{1}{4 \pi}\left(\frac{\bar{y}_{111}^{\overline{R R}} \bar{z}_{112}^{R R}}{m_{\bar{S}_{1}}^{2}}\right)^{2} \frac{\left|V_{u d}\right|^{2}\left|V_{u s}\right|^{2}}{m_{s}^{2}} \frac{G_{F}^{2} f_{\pi}^{2} m_{\pi^{+}}^{4}}{\left(m_{u}+m_{d}\right)^{2}} \\
& \times \alpha_{L}^{2} \frac{m_{p}^{2}-m_{\pi^{+}}^{2}+m_{\chi}^{2}}{m_{p}^{3}} \lambda^{1 / 2}\left(m_{\pi^{+}}^{2}, m_{p}^{2}, m_{\chi}^{2}\right) .
\end{aligned}
$$

Here the parameter $\alpha_{L}$ is defined as $P_{R} u_{p} \alpha_{L}=$ $\epsilon^{i j k}\left\langle 0\left|\bar{u}_{L i}^{c} u_{L j} d_{R k}\right| p\right\rangle \quad$ (see, e.g., [37]) with $\alpha_{L}=$ $0.0100(12)(214) \mathrm{GeV}^{3}$ obtained by the lattice calculation [38], $f_{\pi}=0.13 \mathrm{GeV}$. We do not discuss loop induced $N \rightarrow \pi \chi$, due to the additional suppression by the loop factor $1 /\left(16 \pi^{2}\right)$ as explained in [35].

It is instructive to determine the suppression factor for the decay widths of $p \rightarrow \pi^{+} \chi$ and $p \rightarrow K^{+} \chi$ in the case of $\bar{S}_{1}$ with $m_{\chi}=0.443 \mathrm{GeV}$ (see Figs. 3, 4)

$$
\left.\frac{\Gamma\left(p \rightarrow \pi^{+} \chi\right)}{\Gamma\left(p \rightarrow K^{+} \chi\right)}\right|_{\bar{S}_{1}} \sim 10^{-10}
$$

and in the case of the same processes induced by $S_{1}$ (see Figs. 1 and 2)

$$
\left.\frac{\Gamma\left(p \rightarrow \pi^{+} \chi\right)}{\Gamma\left(p \rightarrow K^{+} \chi\right)}\right|_{S_{1}} \sim 10^{-1}
$$

In the case of $S_{1}$ one can derive bound

$$
\frac{y_{111}^{\overline{R R}} z_{111}^{R R}}{M_{S_{1}}^{2}} \leq 2.83 \times 10^{-30} \mathrm{GeV}^{-2} .
$$

In the case of $\bar{S}_{1}$, one has the same value for $y_{111}^{\overline{R R}} \bar{z}_{111}^{R R} / M_{\bar{S}_{1}}^{2}$, both determined for $m_{\chi}=0.443 \mathrm{GeV}$. Obviously, with the improved precision in searches of proton decays, finding $p \rightarrow K \chi$ and not seeing $p \rightarrow \pi \chi$ would be a possible signature of $\bar{S}_{1}$ mediation in nucleon decays. The same processes mediated by $S_{1}$ does not follow that pattern, differing only by one order of magnitude. One might wonder if neutron can decay into pseudoscalar meson and invisible fermion while proton cannot. In the case of kaons in the final state that is not possible due to $m_{K^{+}}=$ $0.4937 \mathrm{GeV}$ being smaller than $m_{K^{0}}=0.4976 \mathrm{GeV}$. In the pionic case $m_{\pi^{+}}=0.13957 \mathrm{GeV}$ larger than $m_{\pi^{0}}=$ $0.13497 \mathrm{GeV}$. One would think that mass of the invisible fermion should be larger than $m_{p}-m_{\pi^{+}}$, which then kinematically forbids the decay $p \rightarrow \pi^{+} \chi$ and allows $n \rightarrow \pi^{0} \chi$. However, in both $S_{1}$ and $\bar{S}_{1}$ cases, one can construct the dimension-nine operator, which will allow decays $p \rightarrow \chi e^{+} \nu$ forcing $S_{1}\left(\bar{S}_{1}\right)$ to have mass of the order of a Grand Unified Theory (GUT) scale. The same mechanism with mass of $m_{\chi}<m_{n}-m_{\eta}$ will imply $n \rightarrow$ $\eta \chi$ can occur only at the GUT scale.

\section{NEUTRON DECAYS WHILE THE PROTON IS STABLE}

In the case where the mass of invisible fermion is in the range $(937.8,938.8 \mathrm{MeV})$ proton decay is avoided, but neutron transition to $\chi$ is kinematically allowed. The lower bound on the mass of $\chi$ comes from the request that none of the stable nuclei can decay to dark matter, whereas the upper bound is necessary for the stability of $\chi[2,10,17,23]$. In the case of experimental detection, the simplest way is to register photon of the energy $0.782 \mathrm{MeV}<E_{\gamma}<$ $1.664 \mathrm{MeV}$. In order to approach the $n \rightarrow \chi \gamma$ decay amplitude according to [17], one can assume the mixing of $\chi$ and $n$. Following [17], the effective Lagrangian can be written as

$$
\begin{aligned}
\mathcal{L}_{\text {eff }}= & \bar{n}\left(i \not \partial-m_{n}+\frac{g_{n} e}{8 m_{n}} \sigma^{\alpha \beta} F_{\alpha \beta}\right) n \\
& +\bar{\chi}\left(i \partial_{\alpha} \gamma^{\alpha}-m\right) \chi+\epsilon(\bar{n} \chi+\bar{\chi} n),
\end{aligned}
$$

where neutron anomalous magnetic moment is $g_{n}=3.826$ and $\epsilon$ is the mixing parameter with dimension of mass. In the limit $\epsilon \ll m_{n}-m_{\chi}$ [17], one easily finds 


$$
\mathcal{L}_{n \rightarrow \chi \gamma}^{\mathrm{eff}}=\frac{g_{n} e}{8 m_{n}} \frac{\epsilon}{m_{n}-m_{\chi}} \bar{\chi} \sigma^{\alpha \beta} F_{\alpha \beta} n
$$

In the case considered by [17], the decay $n \rightarrow \chi \gamma$ occurs at the tree level with the mediation of the colored scalar $(\overline{3}, 1,1 / 3)$. However, the $\bar{S}_{1}$ colored scalar can mediate such process only at the loop level. Actually, it has to be a box diagram with one $\bar{S}_{1}$ and one $W$ (see Fig. 1) for the $n \rightarrow \chi$ transition. In principle, there is a possibility that in the case of $u \chi \rightarrow s^{c}\left(b^{c}\right) \bar{d}$ process, the $s(b)$ quark is transformed to $d$ while the uplike quark and $W$ are mediated in the loop. However, these contributions are suppressed by the mass of $d$ quark and Glashow-Iliopoulos-Maiani (GIM) mechanism and can therefore be neglected.

A contribution of the box diagram to the $n \rightarrow \chi$ decay amplitude is presented in Fig. 5. Instead of $\epsilon$, in (16) we use $\bar{\epsilon}$ for the mediator $\bar{S}_{1}$

$$
\begin{aligned}
\bar{\varepsilon}= & \alpha_{L} \frac{8 G_{F}}{\sqrt{2}} \sum_{D=s, b} \sum_{U=u, c, t} \bar{y}_{1 U j}^{\overline{R R}} \bar{z}_{1 d D}^{R R} V_{U d} V_{u D}^{*} \\
& \times m_{D} m_{U} I\left(x_{U}, x_{D}, M_{\bar{S}_{1}}\right),
\end{aligned}
$$

with the integral

$$
\begin{aligned}
& I\left(x_{1}, x_{2}, x_{\bar{S}_{1}}\right) \\
& =\frac{1}{64 m_{W}^{2} \pi^{2}}\left[\frac{\left(4-x_{1}\right) x_{1} \ln x_{1}}{\left(1-x_{1}\right)\left(x_{1}-x_{2}\right)\left(x_{1}-x_{\bar{S}_{1}}\right)}\right. \\
& \quad-\frac{\left(-4+x_{2}\right) x_{2} \ln x_{1}}{\left(1-x_{1}\right)\left(x_{1}-x_{2}\right)\left(x_{2}-x_{\bar{S}_{1}}\right)} \\
& \left.\quad+\frac{\left(-4+x_{\bar{S}_{1}}\right) x_{\bar{S}_{1}} \ln x_{\bar{S}_{1}}}{\left(1-x_{1}\right)\left(x_{1}-x_{\bar{S}_{1}}\right)\left(x_{2}-x_{\bar{S}_{1}}\right)}\right] .
\end{aligned}
$$

In this expression $x_{i}=m_{i}^{2} / m_{W}^{2}$.

The dominant contribution from the box diagram comes from the $(c, b)(t, b)$ and $(c, s)$ quarks mediated in the box. In the box diagram in Fig. 5, the up quarks interact with the colored scalar $\bar{S}_{1}$. The downlike couplings to $\bar{S}_{1}$ can be constrained using the oscillations of $K^{0}-\bar{K}^{0}, B_{d, s}^{0}-\bar{B}_{d, s}^{0}[14,40]$. Note that the couplings in the interacting Lagrangian (1) are antisymmetric, which prevents tree-level contributions to these processes. In Appendix A, we present box diagram contributions to the transitions of $K^{0}-\bar{K}^{0}, B_{d, s}^{0}-\bar{B}_{d, s}^{0}$ and determine bounds on the interactions of $\bar{S}_{1}$ with the down

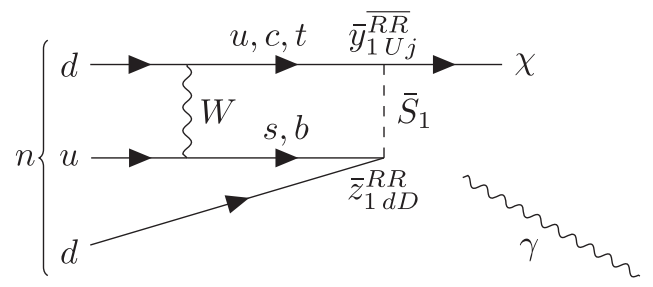

FIG. 5. The box diagram contributing to $n \rightarrow \chi \gamma$. There are also contributions of the crossed diagram.

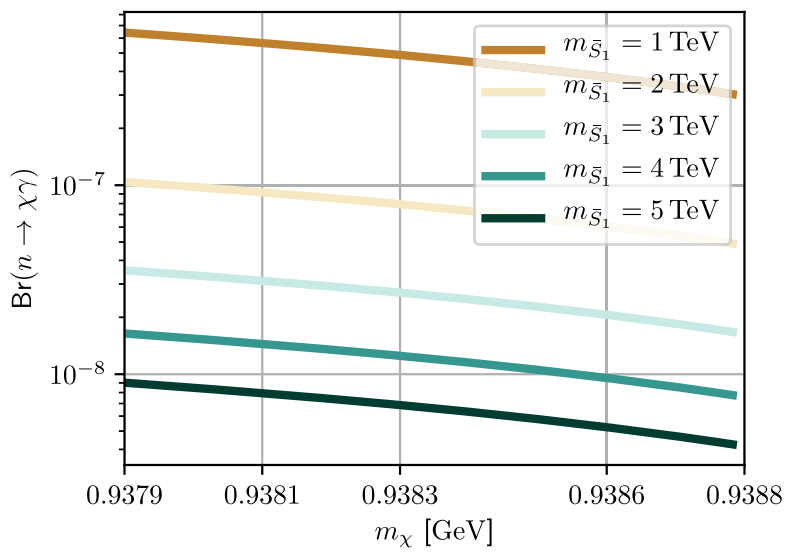

FIG. 6. Branching ratio for $n \rightarrow \chi \gamma$ as a function of $m_{\chi}$ for the different masses of $\bar{S}_{1}$ with $\bar{y}_{11 j} \overline{R R} \simeq \sqrt{4} \pi, j=2,3$.

quarks. Here we give bounds on the couplings we use in our calculation: $\left|\bar{z}_{132}^{R R}\right| \leq 9.21\left(M_{\bar{S}_{1}} / \mathrm{GeV}\right)^{1 / 2} 10^{-4},\left|\bar{z}_{131}^{R R}\right| \leq$ $4.18\left(M_{\bar{S}_{1}} / \mathrm{GeV}\right)^{1 / 2} 10^{-3}$ and $\left|z_{112}^{R R}\right| \leq 0.028\left(M_{\bar{S}_{1}} / \mathrm{GeV}\right)^{1 / 2}$.

The neutron invisible decay width is given by [17]

$$
\Delta \Gamma_{n \rightarrow \chi \gamma}=\frac{g_{n}^{2} e^{2}}{128 \pi} \frac{m_{n} \bar{\varepsilon}^{2}}{\left(m_{n}-m_{\chi}\right)^{2}}\left(1-\frac{m_{\chi}^{2}}{m_{n}^{2}}\right)^{3} .
$$

According to [17], the branching fraction of neutron decay to invisible fermion and photon should be $1 \%$ to explain the neutron lifetime anomaly. In their case the parameter is $\epsilon=\beta y_{111}^{\overline{R R}} z_{111}^{R R} / m_{S_{1}}^{2}\left(S_{1}\right.$ corresponds to $\phi$ in [17]). The parameter $\beta=0.0144(3)(21) \mathrm{GeV}^{3}$ [38] requires that the branching ratio for $n \rightarrow \chi \gamma$ is of the order 1\%. They obtained that $y_{111}^{\overline{R R}} z_{111}^{R R} / m_{S_{1}}^{2} \sim 8 \times 10^{-6} \mathrm{TeV}^{-2}$. Note that for $m_{S_{1}} \sim 1 \mathrm{TeV}$ the product of $y_{111}^{\overline{R R}} z_{111}^{R R} \leq 10^{-6}$.

In Fig. 6 we present dependence of the branching ratio $\operatorname{Br}(n \rightarrow \chi \gamma)$ on the mass of $\chi$ for a given $\bar{S}_{1}$ mass. It is interesting that for the mass of $M_{\bar{S}_{1}}=1 \mathrm{TeV}$ the branching

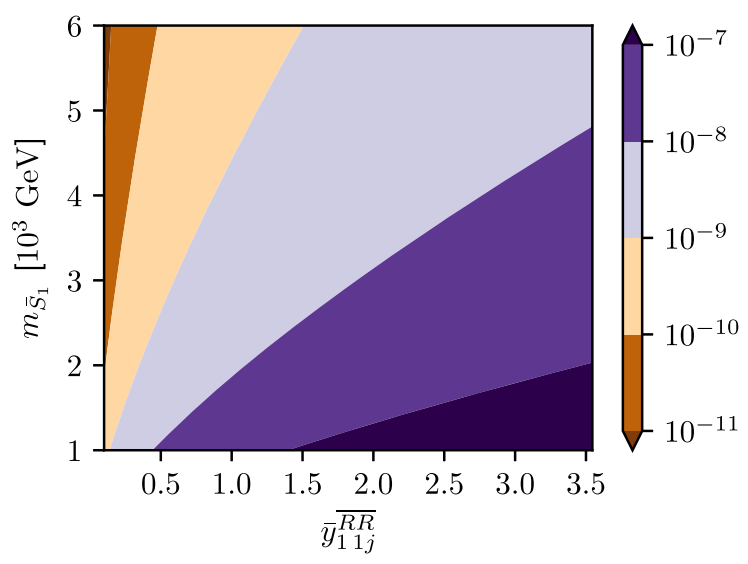

FIG. 7. Branching ratio for $n \rightarrow \chi \gamma$ as a function of $M_{\bar{S}_{1}}$ with $\bar{y}_{112}^{\overline{R R}} \simeq \bar{y}_{113}^{\overline{R R}}$. 


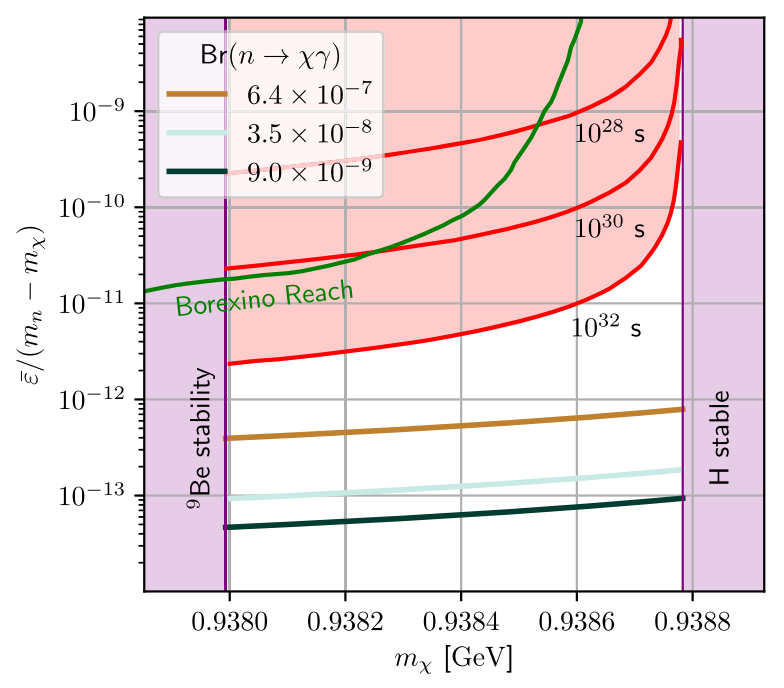

FIG. 8. The values $\bar{\epsilon} /\left(m_{n}-m_{\chi}\right)$ that yield the neutron decay $n \rightarrow \chi \gamma$ as a function of $m_{\chi}$ for different values of the branching ratio $\operatorname{Br}(n \rightarrow \chi \gamma)$. The violet regions are excluded by experiment ${ }^{9} \mathrm{Be}$ and $\mathrm{H}$ stability [2]. The red contours indicate atomic hydrogen lifetimes of $10^{28} \mathrm{~s}, 10^{30} \mathrm{~s}$, and $10^{32} \mathrm{~s}$ obtained in [2].

ratio is $6.4 \times 10^{-7}$, bellow the Borexino limit as discussed in [2]. The colored scalar $\bar{S}_{1}$ can have a mass within the $\mathrm{TeV}$ regime and is therefore appropriate for the LHC searches.

In Fig. 7 we present branching ratio dependence or the mass $m_{\chi} \mathrm{GeV}$ and allow the couplings $\bar{y}_{112}^{\overline{R R}} \simeq \bar{y}_{113}^{\overline{R R}}$ to be in perturbative regime.

The authors of Ref. [2] explored the data with expectations of solar neutrinos and backgrounds from radioactivity to derive bounds on the neutron-mixing parameter $\bar{\epsilon} /\left(m_{n}-m_{\chi}\right)$. They expressed the upper limits on the number of events as lower limits on the $\mathrm{H}$ lifetime are $10^{28} \mathrm{~s}, 10^{30} \mathrm{~s}$, and $10^{32} \mathrm{~s}$ (see Fig. 8). The green line is the $90 \%$ C.L. lower limit from their fit procedure to Borexino data.

The values of parameter $\bar{\epsilon} /\left(m_{n}-m_{\chi}\right)$, coming from the calculation of $n-\chi$ oscillations, are allowed by the analysis of [2] and the mass of $\bar{S}_{1}$ can be reached by LHC. In particular, the decay of $\bar{S}_{1}$ to two jets and $\bar{S}_{1} \rightarrow c(t) \chi$ (monojet) studies were already done by the authors of [29] for larger masses of $\chi$, than the ones we use in this paper.

\section{POSSIBLE LOW-ENERGY SIGNATURES}

The processes in which upper quarks couple to an invisible fermion $\chi$ might offer possible experimentally interesting signatures. Here we consider low-energy decays at the tree level induced by $\bar{S}_{1}$ with $\chi$ in the final state. These decays have invisible fermions in the final state with mass $m_{\chi} \simeq 0.938 \mathrm{GeV}$, allowed by the decay of neutron $n \rightarrow \chi \gamma$, leaving the proton stable. We comment on the loop-level decay $b \rightarrow s \chi \bar{\chi}$. The coupling of top quark with $\chi$ and $\bar{S}_{1}$ can be nonzero, making a search for $t$ to two jets and invisible particle possible. However, it will be very difficult to distinguish such a signal from the decays of top to two jets at LHC.

\section{A. $\Lambda \rightarrow \chi \gamma$}

Assuming nonzero coupling of $\chi$ to $u$ quark $\left(\bar{y}_{111}^{R R} \neq 0\right)$ one can generate oscillations of the $\Lambda$ baryon to $\chi$ as presented in Fig. 9. By a simple replacement of $n$ by $\Lambda$ states and $g_{n}$ by $g_{\Lambda}$ in Eq. (20), one can write

$$
\begin{aligned}
\mathcal{L}_{\text {eff }}(\Lambda)= & \bar{\Lambda}\left(i \not \partial-m_{\lambda}+\frac{g_{\lambda} e}{8 m_{\lambda}} \sigma^{\alpha \beta} F_{\alpha \beta}\right) \lambda \\
& \left.+\bar{\chi}\left(i \partial_{\alpha} \gamma^{\alpha}-m\right) \chi\right)+\epsilon_{\Lambda}(\bar{\lambda} \chi+\bar{\chi} \lambda),
\end{aligned}
$$

leading to the decay width

$$
\Delta \Gamma_{\Lambda \rightarrow \chi \gamma}=\frac{g_{\Lambda}^{2} e^{2}}{128 \pi} \frac{m_{\Lambda} \bar{\varepsilon}_{\Lambda}^{2}}{\left(m_{\Lambda}-m_{\chi}\right)^{2}}\left(1-\frac{m_{\chi}^{2}}{m_{\Lambda}^{2}}\right)^{3} .
$$

where $\bar{\varepsilon}_{\Lambda}=\beta_{\Lambda}\left(\bar{y}_{111}^{R R} \bar{z}_{112}^{R R}\right) / M_{\bar{S}_{1}}^{2}$. We use $g_{\Lambda}=-1.22$ as given in [41] and assume that the $S U(3)$ flavor symmetry holds. Then, the matrix element $\left\langle 0\left|\epsilon_{\rho \sigma \kappa}\left(\bar{u}_{L \rho}^{c} d_{R \sigma} s_{R \kappa}\right)\right| \Lambda\right\rangle$ is not very different from the matrix element for the neutron, $\beta_{\Lambda} \simeq \beta=0.0144(3)(21) \mathrm{GeV}^{3}$ [37]. Current experimental limits on the rates for the baryon number violating processes $\Lambda \rightarrow \pi^{+} e, \Lambda \rightarrow \pi^{+} \mu^{-}$are smaller than $6 \times 10^{-7}$ $[41,42]$ and for other searched channel the bounds are even weaker. Using Eq. (22), it is easy to calculate

$$
\left.\operatorname{Br}(\Lambda \rightarrow \chi \gamma)\right|_{M_{\bar{S}_{1}}=5 \mathrm{TeV}}=\left|\bar{y}_{111}^{\overline{R R}}\right|^{2} 1.75 \times 10^{-6} .
$$

Obviously that such bound would require $\bar{y}_{111}^{\overline{R R}} \ll 1$. It seems that the coupling of the $u$ quark to the invisible fermion should be very suppressed. From a number of cases studied in the literature (see, e.g., $[14,43]$ ), the couplings of the first quark generation to leptons and leptoquarks are very suppressed compared to the other two generations. Using the constraint from $D^{0}-\bar{D}^{0}$ oscillations (see Appendix B) we notice, that the product is $\bar{y}_{111}^{\overline{R R}} \bar{y}_{121}^{\overline{R R} *}<$ $1.1 \times 10^{-5} M_{\bar{S}_{1}} / \mathrm{GeV}$. Requirement that $\bar{y} \overline{111}$ has to be small, leaves a possibility that the coupling $\bar{y}_{121}^{\overline{R R} *}$ can be of the order 1 . This is exactly what is necessary for our

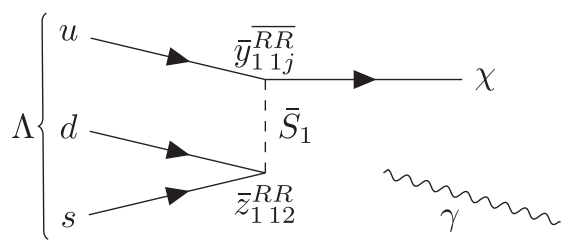

FIG. 9. $\Lambda \rightarrow \chi \gamma$. 
analysis of $n \rightarrow \chi \gamma$. Obviously, if the $\Lambda \rightarrow \chi \gamma$ decay is forbidden, the coupling of $u$ quark should be set to zero.

\section{B. Heavy hadron decays to invisibles}

For the mass $m_{\chi} \simeq 0.938 \mathrm{GeV}$, decays of charmed mesons to invisible fermions are not allowed kinematically. However, baryons containing one $c$ quark and two light quark, e.g., $\Lambda_{c}^{+}$or $\Sigma_{c}^{0}$ can decay to invisible fermions. The processes as $\Lambda_{c}^{+} \rightarrow K^{+} \chi$ and $\Sigma_{c}^{0} \rightarrow \chi \gamma$ are allowed. Using Eq. (8), assuming that the matrix element of $\left\langle K^{+}\left|\epsilon_{\alpha \beta \gamma}\left(c_{\alpha}^{T} C P_{\Gamma} d_{\beta}\right) P_{\Gamma^{\prime}} s_{\gamma}\right| \Lambda_{c}^{+}\right\rangle$is not very different from the one in Eq. (6), using Particle Data Group (PGD) data for the relevant parameters [41] we estimate that $\operatorname{Br}\left(\Lambda_{c}^{+} \rightarrow\right.$ $\left.K^{+} \chi\right)<10^{-6}$ (for $\bar{y}_{121}^{\overline{R R} *} \simeq 1$ and $M_{\bar{S}_{1}} \sim 2 \mathrm{GeV}$ ). The $\Sigma_{c}$ can decay to $\chi \gamma$. Taking the anomalous magnetic moment of $\Sigma_{c}^{0}$ to be $\simeq-2.7$, as calculated in [44], we obtain, by appropriate replacements in Eq. (20), that the rate for $\Sigma_{c}^{0} \rightarrow$ $\chi \gamma$ is very suppressed, being in the order of $10^{-16}$, making it impossible to be seen.

Possible decays of heavy hadrons with baryon number violations were discussed in $[8,45]$. The decay $B^{+} \rightarrow \Lambda_{c} \chi$ will be allowed within our approach, however very suppressed if the same assumptions as in [45] are used. On the experimental side, there are more searches. For example in BESSIII [46] they search for the processes $D^{+} \rightarrow \bar{\Lambda}\left(\bar{\Sigma}^{0}\right) e^{+}$ and $D^{+} \rightarrow \Lambda\left(\Sigma^{0}\right) e^{+}$, for which the upper limits on the branching fractions are set at the level of $\mathcal{O}\left(10^{-6}\right)$. Kinematics forbid $D^{0} \rightarrow \bar{\Lambda} \chi$ decay for the mass of $\chi$ being close to the nucleon mass. Due to the lack of lattice QCD result on the matrix elements $\left\langle\bar{\Lambda}\left|\epsilon_{\alpha \beta \gamma}\left(c_{\alpha}^{*} C P_{\Gamma} d_{\beta}^{\dagger}\right) P_{\Gamma^{\prime}} s_{\gamma}^{\dagger}\right| D^{0}\right\rangle$, one can assume they are close in value to the one in Eq. (6). Even if we take $m_{\chi}=0.443 \mathrm{GeV}$, our rough estimate leads to the branching ratio $\operatorname{Br}\left(D^{0} \rightarrow \Lambda \chi\right) \leq 10^{-19}$, making it too small to be measured.

\section{1. $J / \psi \rightarrow \chi \bar{\chi}$}

The dominant contribution to $\Delta \Gamma(n \rightarrow \chi \gamma)$ induced by $\bar{S}_{1}$ comes from the coupling of $c$ quark to $\chi$. One would immediately suggest that the $c \bar{c}$ bound state might decay to two invisible fermions. Only the lower bound $\mathrm{BR}(J / \psi \rightarrow$ invisibles) $<7 \times 10^{-4}$ is experimentally known.

The amplitude for decay $J / \psi \rightarrow \chi \bar{\chi}$ at the tree level in Fig. 10 can be obtained using the effective Lagrangian approach as in [14]

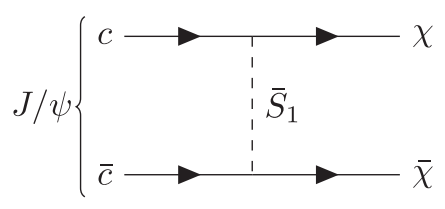

FIG. 10. $J / \psi$ decay to invisibles.

$$
\mathcal{L}_{\text {eff }}=\sqrt{2} G_{F} \frac{v^{2}}{2 M_{\bar{S}_{1}}^{2}}|\bar{y} \overline{R R}|^{2}\left(\bar{c} \gamma_{\mu} P_{R} c\right)\left(\bar{\chi} \gamma^{\mu} P_{R} \chi\right) .
$$

By introducing $\left\langle 0\left|\bar{c} \gamma_{\mu} c\right| J / \psi(\epsilon, P)\right\rangle=f_{J \psi} m_{J / \psi} \epsilon_{\mu}$ [47], the decay width is given by

$$
\Gamma(J / \psi \rightarrow \chi \bar{\chi})=\frac{f_{J \psi}^{2}}{2 \pi m_{J \psi}}\left(1-4 x_{\chi}^{2}\right)^{1 / 2}\left(1-x_{\chi}^{2}\right)|\mathcal{A}|^{2}
$$

with $\mathcal{A} \equiv \sqrt{2} G_{F} \frac{v^{2}}{2 M_{J / \psi}^{2}}\left|\bar{y}_{121}^{R R}\right|^{2}$ and $x_{\chi}=m_{\chi} / M_{J / \psi}$. The experimental bound is very week, allowing huge $\bar{y}_{112}^{\overline{R R}}$ coupling. For $m_{\chi}=0.938 \mathrm{GeV}$ and $M_{\bar{S}_{1}}$ given in $\mathrm{TeV}$, branching ratio is

$$
\operatorname{Br}(J / \psi \rightarrow \chi \bar{\chi}) \leq \frac{\left|y_{112}^{\overline{R R}}\right|^{4}}{M_{\bar{S}_{1}}^{4}} \mathrm{TeV}^{4} \times 10^{-7} .
$$

This is three orders of magnitude smaller than the current experimental result in [41].

$$
\text { 2. } b \rightarrow s \chi \bar{\chi}
$$

The amplitude for $b \rightarrow s \chi \bar{\chi}$ comes from the contributions presented in Fig. 11. and equals to

$$
\begin{aligned}
\mathcal{M}(b \rightarrow s \chi \bar{\chi}) & =\frac{8 G_{F}}{\sqrt{2}} \sum_{i, j=c, t} \bar{y}_{1 i 1}^{\overline{R R}} \bar{y}_{1 j 1}^{\overline{R R} *} V_{i b} V_{j s}^{*} \\
& \times m_{i} m_{j}\left(\bar{s} \gamma^{\mu} P_{L} b\right)\left(\bar{\chi} \gamma_{\mu} P_{R} \chi\right) I\left(x_{i}, x_{j}, x_{\bar{S}_{1}}\right) .
\end{aligned}
$$

If we compare the appropriate Wilson coefficient for the $b \rightarrow s \chi \bar{\chi}$ and the numerical value for $M_{\bar{S}_{1}} \sim 1 \mathrm{TeV}$, we obtain that it is more than two orders of magnitude suppressed compared to the Wilson coefficient for the SM transition $b \rightarrow s \nu \bar{\nu}$ calculated in [48]. This makes the invisible fermion search in the exclusive processes $B \rightarrow K^{(*)} \chi \bar{\chi}$ very difficult. The decays of $B \rightarrow K^{(*)} \chi \bar{\chi}$ were considered in Ref. [49] for the mass of invisible fermions kinematically allowed.

\section{Possible color scalar signatures at LHC}

A proposal to search for signals of colored scalars was made in [50], based on interactions of colored scalars with

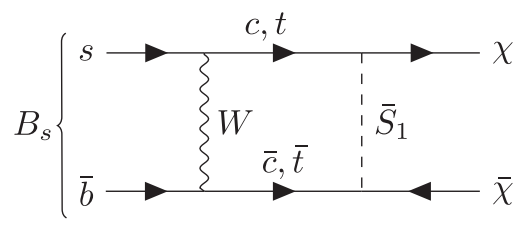

FIG. 11. $B_{s} \rightarrow \chi \bar{\chi}$. 
up (down) quarks couplings to invisible. The LHC search for colored scalar with the couplings we consider in this paper, would potentially be performed in the final states containing two light quark jets and monojet and missing energy. The authors of Ref. [51] followed the proposal of Ref. [40] and, using the data of [52], derived new bounds for the couplings of color triplet scalars to two up- (down) like quarks, which were improved by almost two orders of magnitude for light quark jets. However, dijet couplings are still better constrained by meson oscillations. Hopefully, further LHC searches, such as that of CMS [53], will improve the limits for the model with such particular couplings.

\section{SUMMARY}

Invisible right-handed fermions can appear in different theoretical frameworks. Here we consider a model in which a colored scalar $\bar{S}_{1}=(\overline{3}, 1,-2 / 3)$ couples either to uplike quarks and invisible right-handed fermion or two downlike quarks of different flavor species. In the case that both proton and neutron are unstable, decays of $N \rightarrow K \chi$ are possible with mass of $\bar{S}_{1}$ at GUT scale. The neutron can decay to $n \rightarrow \pi^{0} \chi$ for the mass of $0.7987 \mathrm{GeV}<m_{\chi}<$ $0.8045 \mathrm{GeV}$, while decay $p \rightarrow \pi^{-} \chi$ is forbidden at tree level by the dimension-nine operator. However, the dimension-nine operator might induce $p \rightarrow \chi l^{+} \nu_{l}$ with $l=e, \mu$, forcing the mass of $\bar{S}_{1}$ to be at GUT scale.

In the case when the neutron decays and the proton is stable, the mass of $\chi$ has a very narrow range. The $\bar{S}_{1}$ can mediate $n \rightarrow \chi \gamma$ at loop level with mass of colored scalar $\bar{S}_{1}$ of the order TeV scale, appropriate for the LHC searches. The contributions of $c$ and $t$ coupling to $\chi$ are largest in this case. The decay rate of $n \rightarrow \chi \gamma$ can reach $\sim 10^{-6}$, which is in agreement with the Borexino experiment bound. Further searches of such decays by KamLand and other experiments would help to distinguish between the models of invisible fermions. An interesting proposal to search for invisible fermions by their capture by atomic nuclei was done in Ref. [23] suggesting that the large volume neutrino experiments can be used for such searches. This opens up new possibility for searches at DUNE, and at various xenon experiment as explained by the authors [23].

Further, we searched for possible signatures of the fermionic invisible particles, coupling to up quarks via $\bar{S}_{1}$ and found that at tree level one can produce $\Lambda \rightarrow \chi \gamma$ decay. Obtaining the experimental bound on such decay rate would be very important for the model presented in this paper as well as for obtaining the constraint on the $u$ quark coupling to $\chi$. Search for $J / \psi \rightarrow \chi \bar{\chi}$ would shed more light on the possible charm quark coupling to invisible fermions. There are ongoing searches at LHC which will shed more light on the eventual existence of colored scalars.

\section{ACKNOWLEDGMENTS}

The work of S. F. was in part financially supported by the Slovenian Research Agency (research core funding Grant No. P1-0035). The work of D. S. was financially supported by the Slovenian Research Agency (research core funding Grant No. PR-10495). We are grateful to Darius Faroughy, Damir Bečirević, and Nejc Košnik for very insightful discussions.

\section{APPENDIX A: DIQUARK COUPLINGS}

The contributions from the diquark couplings in Lagrangian (1) appear in the oscillations of $B_{s}-\bar{B}_{s}$, $B_{d}-\bar{B}_{d}$ and $K^{0}-\bar{K}^{0}$ mesons (see Fig. 12). In the case of $B_{s}-\bar{B}_{s}$, there are contributions from the two box diagrams with $d$ quarks within the box. In the case of $B_{d}-\bar{B}_{d}\left(K^{0}-\bar{K}^{0}\right)$, internal $s(b)$ quarks contribute. The couplings $\left(\bar{z}_{1}\right)_{i j}$ are antisymmetric $\left(\left(\bar{z}_{1}\right)_{i j}=-\left(\bar{z}_{1}\right)_{j i}\right)$. The contributions of $\bar{S}_{1}$ box diagrams in the case of the $B_{s}-\bar{B}_{s}$ oscillation are

$\mathcal{L}_{\Delta B=2}^{\mathrm{NP}}=-\frac{1}{128 \pi^{2}} \frac{\left(\bar{z}_{113}^{R R}\right)^{2}\left(\bar{z}_{123}^{R R}\right)^{* 2}}{M_{\bar{S}_{1}}^{2}}\left(\bar{s} \gamma_{\mu} P_{R} b\right)\left(\bar{s} \gamma^{\mu} P_{R} b\right)$.

This result can be understood in terms of the recent study of new physics in the $B_{s}-\bar{B}_{s}$ oscillation in [54]. The authors of [54] introduced the following notation of the NP contribution containing the right-handed operators as

$\mathcal{L}_{\Delta B=2}^{\mathrm{NP}} \supset-\frac{4 G_{F}}{\sqrt{2}}\left(V_{t b} V_{t s}^{*}\right)^{2} C_{b s}^{R R}\left(\bar{s} \gamma_{\mu} P_{R} b\right)\left(\bar{s} \gamma^{\mu} P_{R} b\right)$.

Following their notation, one can write the modification of the SM contribution by the NP as in Ref. [54]

$$
\frac{\Delta M_{s}^{\mathrm{SM}+\mathrm{NP}}}{\Delta M_{s}^{\mathrm{SM}}}=\left|1+\frac{\eta^{6 / 23}}{R_{\mathrm{loop}}^{\mathrm{SM}}} C_{b s}^{R R}\right|
$$

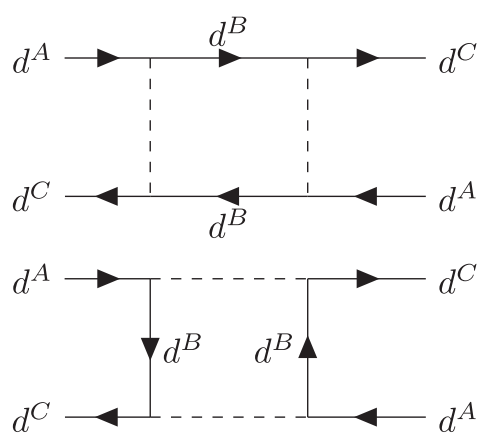

FIG. 12. The diagrams showing oscillations of mesons consisting of down quark and down antiquark. The $d^{A}$ for $A=1,2,3$ corresponds $d, s, b$ quarks. 
They found that $R_{\text {loop }}^{\mathrm{SM}}=(1.31 \pm 0.010) \times 10^{-3}$ and $\eta=\alpha_{s}\left(\mu_{\mathrm{NP}}\right) / \alpha_{s}\left(\mu_{b}\right)$. Relying on the Lattice QCD results of the two collaborations FNAL/MILC [55], HPQCD [56], the FLAG averaging group [57] published following results, which we use in our calculations

$$
\begin{aligned}
& \Delta M_{s}^{\mathrm{FLAG} 2019}=\left(20.1_{-1.6}^{+1.2}\right) \mathrm{ps}^{-1}=\left(1.13_{-0.09}^{+0.07}\right) \Delta M_{s}^{\exp }, \\
& \Delta M_{d}^{\text {FLAG2019 }}=\left(0.582_{-0.056}^{+0.049}\right) \mathrm{ps}^{-1}=\left(1.15_{-0.11}^{+0.10}\right) \Delta M_{d}^{\exp } .
\end{aligned}
$$

From these results, one can easily determine bound

$$
\frac{\left(\bar{z}_{121}^{R R}\right)^{2}\left(\bar{z}_{131}^{R R}\right)^{* 2}}{M_{\bar{S}_{1}}^{2}} \leq 1.17 \times 10^{-4} \mathrm{GeV}^{-2},
$$

while in the case of $B_{d}-\bar{B}_{d}$, following the procedure of [54], by appropriate replacements $s \leftrightarrow d$, the constraint is

$$
\frac{\left(\bar{z}_{121}^{R R}\right)^{2}\left(\bar{z}_{132}^{R R}\right)^{* 2}}{M_{\bar{S}_{1}}^{2}} \leq 2.58 \times 10^{-5} \mathrm{GeV}^{-2} .
$$

Following the work of $[31,58,59]$ for the treatment of $K^{0}-\bar{K}^{0}$, we consider

$$
M_{12}^{K}=\frac{1}{2 m_{K}}\left\langle\bar{K}^{0}\left|\mathcal{H}_{\mathrm{eff}}^{\Delta S=2}\right| K^{0}\right\rangle .
$$

As discussed in $[58,59]$ the short distance SM value for $M_{12}^{K}$ is found to be

$$
M_{12}^{K, \mathrm{SM}}=\frac{G_{F}^{2}}{12 \pi^{2}} f_{K}^{2} B_{K} m_{K} m_{W} F_{0}\left(x_{c}, x_{t}\right),
$$

with the function $F_{0}\left(x_{c}, x_{t}\right)=\lambda_{c}^{2} \eta_{c c} S_{0}(x)+\lambda_{t}^{2} \eta_{t t} S_{0}(y)+$ $2 \lambda_{c} \lambda_{t} \eta_{c t} S_{0}(x, y) . B_{K}$ is a bag parameter and $f_{K}$ is kaon decay constant. They are all introduced in $[58,59]$. The effective Lagrangian can be straightforwardly derived by appropriate replacement in Eq. (A2).

Such Lagrangian gives the following contribution to $M_{K, 12}^{\bar{S}_{1}}$

$$
M_{K, 12}^{\bar{S}_{1}}=\frac{\left(\bar{z}_{131}^{R R}\right)^{2}\left(\bar{z}_{132}^{R R}\right)^{* 2}}{M_{\bar{S}_{1}}^{2}} \frac{1}{192 \pi^{2}} m_{K}^{2} \hat{B}_{K} \eta^{2}
$$

The values are $\hat{B}_{K}=0.727, m_{K}=0.4976$ and $\eta_{2}=0.58$ as in $[27,60]$. This leads to

$$
\frac{\left(\bar{z}_{131}^{R R}\right)^{2}\left(\bar{z}_{132}^{R R}\right)^{* 2}}{M_{\bar{S}_{1}}^{2}} \leq 3.85 \times 10^{-6} \mathrm{GeV}^{-2}
$$

Using these constraints, one can find $\left|\bar{z}_{132}^{R R}\right| \leq 9.21 \times$ $10^{-4} \sqrt{M_{\bar{S}_{1}} / \mathrm{GeV}}, \quad\left|\bar{z}_{131}^{R R}\right| \leq 4.18 \times 10^{-3} \sqrt{M_{\bar{S}_{1}} / \mathrm{GeV}}$ and $\left|z_{112}^{R R}\right| \leq 0.028 \sqrt{M_{\bar{S}_{1}} / \mathrm{GeV}}$.

\section{APPENDIX B: CONSTRAINTS FROM $D^{0}-\bar{D}^{\mathbf{0}}$}

The effective Hamiltonian describing the $D^{0}-\bar{D}^{0}$ oscillation is $\mathcal{H}=C_{6}\left(\bar{u}_{R} \gamma C_{R}\right)\left(\bar{u}_{R} \gamma C_{R}\right)$. The effective Wilson coefficient in the case when two $\chi$ and two $\bar{S}_{1}$ are exchanged within the box, one can easily calculate

$$
C_{6}\left(M_{\bar{S}_{1}}\right)=-\frac{\bar{y}_{111}^{\overline{R R} 2} \bar{y}_{11}^{\overline{R R} * 2}}{64 \pi^{2} M_{\bar{S}_{1}}^{2}} .
$$

Usually, the hadronic matrix element $\left\langle\bar{D}^{0}\right|\left(\bar{u}_{R} \gamma C_{R}\right) \times$ $\left(\bar{u}_{R} \gamma C_{R}\right)\left|D^{0}\right\rangle=\frac{2}{3} m_{D}^{2} F_{D}^{2} B$ with the bag parameter $B_{D}(3 \mathrm{GeV})=0.757(27)(4)$, calculated in the $\overline{\mathrm{MS}}$ scheme, has been computed on the lattice [61]. Due to large nonperturbative contributions, the SM contribution is not well known. Therefore, we can get the robust bound on the product of the couplings by requiring that the mixing frequency, in the absence of $C P$ violation, should be smaller than the world average $x=2\left|M_{12}\right| / \Gamma=\left(0.43_{-0.11}^{+0.10}\right) \%$ as reported by HFLAV [62]. The bound can be obtained as in [63] from

$$
\left|r C_{6}\left(M_{\bar{S}_{1}}\right)\right| \frac{2 m_{D} f_{D}^{2} B_{D}}{3 \Gamma_{D}}<x
$$

where $r=0.76$ is a renormalization factor due to running of $C_{6}$ from scale $M_{\bar{S}_{1}} \simeq 1.5 \mathrm{TeV}$ down to $3 \mathrm{GeV}$. One can easily get $\left|C_{6}\right|<2.2 \times 10^{-13}$ or $\bar{y}_{111}^{\overline{R R}} \bar{y}_{121}^{\overline{R R}}<1.1 \times$ $10^{-5} M_{\bar{S}_{1}} / \mathrm{GeV}$.
[1] J. Heeck, Light particles with baryon and lepton numbers, Phys. Lett. B 813, 136043 (2021).

[2] D. McKeen and M. Pospelov, How long does the hydrogen atom live? arXiv:2003.02270.

[3] J. M. Cline and J. M. Cornell, Dark decay of the neutron, J. High Energy Phys. 07 (2018) 081.
[4] B. Grinstein, C. Kouvaris, and N. G. Nielsen, Neutron Star Stability in Light of the Neutron Decay Anomaly, Phys. Rev. Lett. 123, 091601 (2019).

[5] M. Jin and Y. Gao, Nucleon-Light Dark Matter Annihilation through Baryon Number Violation, Phys. Rev. D 98, 075026 (2018). 
[6] W.-Y. Keung, D. Marfatia, and P.-Y. Tseng, Annihilation signatures of neutron dark decay models in neutron oscillation and proton decay searches, J. High Energy Phys. 09 (2019) 053.

[7] J. M. Cline, M. Puel, and T. Toma, Affleck-Dine inflation, Phys. Rev. D 101, 043014 (2020).

[8] G. Elor, M. Escudero, and A. Nelson, Baryogenesis and dark matter from $B$ mesons, Phys. Rev. D 99, 035031 (2019).

[9] D. Barducci, M. Fabbrichesi, and E. Gabrielli, Neutral hadrons disappearing into the darkness, Phys. Rev. D 98, 035049 (2018).

[10] H. Davoudiasl, Nucleon Decay into a Dark Sector, Phys. Rev. Lett. 114, 051802 (2015).

[11] Z. Berezhiani and L. Bento, Neutron-Mirror Neutron Oscillations: How Fast Might They Be?, Phys. Rev. Lett. 96, 081801 (2006).

[12] Z. Berezhiani, Neutron-antineutron oscillation and baryonic Majoron: Low scale spontaneous baryon violation, Eur. Phys. J. C 76, 705 (2016).

[13] K. Abe et al., Search for Nucleon Decay via $n \rightarrow \bar{\nu} \pi^{0}$ and $p \rightarrow \bar{\nu} \pi^{+}$in Super-Kamiokande, Phys. Rev. Lett. 113, 121802 (2014).

[14] I. Doršner, S. Fajfer, A. Greljo, J. Kamenik, and N. Košnik, Physics of leptoquarks in precision experiments and at particle colliders, Phys. Rep. 641, 1 (2016).

[15] F. del Aguila, S. Bar-Shalom, A. Soni, and J. Wudka, Heavy Majorana neutrinos in the effective lagrangian description: Application to hadron colliders, Phys. Lett. B 670, 399 (2009).

[16] Z. Berezhiani, Neutron lifetime and dark decay of the neutron and hydrogen, Lett. High Energy Phys. 2, 118 (2019).

[17] B. Fornal and B. Grinstein, Dark Matter Interpretation of the Neutron Decay Anomaly, Phys. Rev. Lett. 120, 191801 (2018).

[18] A. Serebrov, Neutron lifetime beam anomaly and possible explanation, arXiv:1912.10006.

[19] M. Tanabashi et al., Review of particle physics, Phys. Rev. D 98, 030001 (2018).

[20] Z. Tang et al., Search for the Neutron Decay $n \rightarrow X+\gamma$ Where $\mathrm{X}$ is a Dark Matter Particle, Phys. Rev. Lett. 121, 022505 (2018).

[21] Z. Berezhiani, Neutron lifetime puzzle and neutron-mirror neutron oscillation, Eur. Phys. J. C 79, 484 (2019).

[22] M. Agostini et al., A Test of Electric Charge Conservation with Borexino, Phys. Rev. Lett. 115, 231802 (2015).

[23] B. Fornal, B. Grinstein, and Y. Zhao, Dark matter capture by atomic nuclei, Phys. Lett. B 811, 135869 (2020).

[24] F. Elahi and M. Mohammadi Najafabadi, Neutron decay to a non-Abelian dark sector, Phys. Rev. D 102, 035011 (2020).

[25] G. Baym, D. Beck, P. Geltenbort, and J. Shelton, Testing Dark Decays of Baryons in Neutron Stars, Phys. Rev. Lett. 121, 061801 (2018).

[26] T. Motta, P. Guichon, and A. Thomas, Implications of neutron star properties for the existence of light dark matter, J. Phys. G 45, 05LT01 (2018).

[27] M. Blanke and S. Kast, Top-flavored dark matter in dark minimal flavor violation, J. High Energy Phys. 05 (2017) 162.
[28] S. Baek, P. Ko, and P. Wu, Heavy quark-philic scalar dark matter with a vector-like fermion portal, J. Cosmol. Astropart. Phys. 07 (2018) 008.

[29] T. Jubb, M. Kirk, and A. Lenz, Charming dark matter, J. High Energy Phys. 12 (2017) 010.

[30] A. Mohamadnejad, Accidental scale-invariant Majorana dark matter in leptoquark-Higgs portals, Nucl. Phys. B949, 114793 (2019).

[31] P. Agrawal, M. Blanke, and K. Gemmler, Flavored dark matter beyond minimal flavor violation, J. High Energy Phys. 10 (2014) 072.

[32] P. B. Dev and R. N. Mohapatra, TeV scale model for baryon and lepton number violation and resonant baryogenesis, Phys. Rev. D 92, 016007 (2015).

[33] A. Goyal and M. Kumar, Fermionic dark matter in a simple $t$-channel model, J. Cosmol. Astropart. Phys. 11 (2016) 001.

[34] R. Allahverdi, P. S. B. Dev, and B. Dutta, A simple testable model of baryon number violation: Baryogenesis, dark matter, neutron-antineutron oscillation and collider signals, Phys. Lett. B 779, 262 (2018).

[35] I. Doršner, S. Fajfer, and N. Košnik, Heavy and light scalar leptoquarks in proton decay, Phys. Rev. D 86, 015013 (2012).

[36] H. Davoudiasl, Gravitationally induced dark matter asymmetry and dark nucleon decay, Phys. Rev. D 88, 095004 (2013).

[37] Y. Aoki, C. Dawson, J. Noaki, and A. Soni, Proton decay matrix elements with domain-wall fermions, Phys. Rev. D 75, 014507 (2007).

[38] Y. Aoki, T. Izubuchi, E. Shintani, and A. Soni, Improved lattice computation of proton decay matrix elements, Phys. Rev. D 96, 014506 (2017).

[39] V. Takhistov et al., Search for Trilepton Nucleon Decay via $p \rightarrow e^{+} \nu \nu$ and $p \rightarrow \mu^{+} \nu \nu$ in the Super-Kamiokande Experiment, Phys. Rev. Lett. 113, 101801 (2014).

[40] G. F. Giudice, B. Gripaios, and R. Sundrum, Flavorful production at hadron colliders, J. High Energy Phys. 08 (2011) 055.

[41] P. Zyla et al., Review of particle physics, Prog. Theor. Exp. Phys. (2020), 083C01.

[42] M. McCracken et al., Search for baryon-number and leptonnumber violating decays of $\Lambda$ hyperons using the CLAS detector at Jefferson Laboratory, Phys. Rev. D 92, 072002 (2015).

[43] I. Doršner, S. Fajfer, and M. Patra, A comparative study of the $S_{1}$ and $U_{1}$ leptoquark effects in the light quark regime, Eur. Phys. J. C 80, 204 (2020).

[44] D. Riska, Physics of charmed baryons and their magnetic moments, Nucl. Instrum. Methods Phys. Res., Sect. B 119, 259 (1996).

[45] W.-S. Hou, M. Nagashima, and A. Soddu, Baryon number violation involving higher generations, Phys. Rev. D 72, 095001 (2005).

[46] M. Ablikim et al., Search for baryon and lepton number violating decays $D^{+} \rightarrow \bar{\Lambda}\left(\bar{\Sigma}^{0}\right) e^{+}$and $D^{+} \rightarrow \Lambda\left(\Sigma^{0}\right) e^{+}$, Phys. Rev. D 101, 031102 (2020).

[47] D. Aloni, A. Efrati, Y. Grossman, and Y. Nir, $\Upsilon$ and $\psi$ leptonic decays as probes of solutions to the $R_{D}^{(*)}$ puzzle, J. High Energy Phys. 06 (2017) 019. 
[48] W. Altmannshofer, A. J. Buras, D. M. Straub, and M. Wick, New strategies for new physics search in $B \rightarrow K^{*} \nu \bar{\nu}, B \rightarrow$ $K \nu \bar{\nu}$ and $B \rightarrow X_{s} \nu \bar{\nu}$ decays, J. High Energy Phys. 04 (2009) 022 .

[49] G. Li, T. Wang, Y. Jiang, J.-B. Zhang, and G.-L. Wang, Spin-1/2 invisible particles in heavy meson decays, Phys. Rev. D 102, 095019 (2020).

[50] D. Abercrombie et al., Dark matter benchmark models for early LHC run-2 searches: Report of the ATLAS/CMS dark matter forum, Phys. Dark Universe 27, 100371 (2020).

[51] B. Pascual-Dias, P. Saha, and D. London, LHC constraints on scalar diquarks, J. High Energy Phys. 07 (2020) 144.

[52] A. M. Sirunyan et al., Search for narrow and broad dijet resonances in proton-proton collisions at $\sqrt{s}=13 \mathrm{TeV}$ and constraints on dark matter mediators and other new particles, J. High Energy Phys. 08 (2018) 130.

[53] A. M. Sirunyan et al., Search for high mass dijet resonances with a new background prediction method in proton-proton collisions at $\sqrt{s}=13 \mathrm{TeV}$, J. High Energy Phys. 05 (2020) 033.

[54] L. Di Luzio, M. Kirk, A. Lenz, and T. Rauh, $\Delta M_{s}$ theory precision confronts flavor anomalies, J. High Energy Phys. 12 (2019) 009.

[55] A. Bazavov et al., $B_{(s)}^{0}$-mixing matrix elements from lattice QCD for the Standard Model and beyond, Phys. Rev. D 93, 113016 (2016).
[56] R. Dowdall, C. Davies, R. Horgan, G. Lepage, C. Monahan, J. Shigemitsu, and M. Wingate, Neutral B-meson mixing from full lattice QCD at the physical point, Phys. Rev. D 100, 094508 (2019).

[57] S. Aoki et al., FLAG review 2019: Flavor lattice averaging group (FLAG), Eur. Phys. J. C 80, 113 (2020).

[58] M. Blanke, A. J. Buras, K. Gemmler, and T. Heidsieck, $\Delta F=2$ observables and $B \rightarrow X_{q} \gamma$ decays in the Left-Right Model: Higgs particles striking back, J. High Energy Phys. 03 (2012) 024.

[59] A. J. Buras and J. Girrbach, Towards the identification of new physics through quark flavor violating processes, Rep. Prog. Phys. 77, 086201 (2014).

[60] M. Bona et al., The UTfit collaboration report on the status of the unitarity triangle beyond the standard model. I. Model-independent analysis and minimal flavor violation, J. High Energy Phys. 03 (2006) 080.

[61] N. Carrasco, P. Dimopoulos, R. Frezzotti, V. Lubicz, G. C. Rossi, S. Simula, and C. Tarantino, $\Delta S=2$ and $\Delta C=2$ bag parameters in the standard model and beyond from $\mathrm{N}_{f}=2+1+1$ twisted-mass lattice QCD, Phys. Rev. D 92, 034516 (2015).

[62] Y.S. Amhis et al., Averages of $b$-hadron, $c$-hadron, and $\tau$-lepton properties as of 2018, arXiv:1909.12524.

[63] S. Fajfer and N. Košnik, Prospects of discovering new physics in rare charm decays, Eur. Phys. J. C 75, 567 (2015). 